\title{
Epidemiology of Klebsiella pneumoniae bloodstream infections in a teaching hospital: factors related to the carbapenem resistance and patient mortality
}

\author{
Lijun $\operatorname{Tian}^{1 \dagger}$, Ruoming $\operatorname{Tan}^{1 \dagger}$, Yang Chen ${ }^{1}$, Jingyong Sun ${ }^{2}$, Jialin Liu ${ }^{1}$, Hongping Qu ${ }^{1 *}$ and Xiaoli Wang ${ }^{1 *}$
}

\begin{abstract}
Background: Although Klebsiella pneumoniae bloodstream infections (KP-BSIs) have recently attracted attention due to an alarming raise in morbidity and mortality, there have been few reports on the epidemiology of KP-BSIs in mainland China. We sought to describe the epidemiological, microbiological, and clinical characteristics of KP-BSIs, focusing on the risk factors of carbapenem resistance and patient mortality.

Methods: A retrospective analysis of WHONET data of KP-BSI patients admitted to a teaching hospital in Shanghai, China, between January 1, 2011 and December 31, 2015 was performed, and the annual percentage of patients with carbapenem-resistant $K$. pneumoniae (CRKP) was determined. Risk factors related to the carbapenem resistance and patient mortality were analyzed using binary logistic regression model. The genetic relatedness of CRKP strains isolated from intensive care unit (ICU) patients was determined by pulsed-field gel electrophoresis (PFGE).

Results: A total of 293 incidences of KP-BSIs were identified in a 5-year period, $22.18 \%$ of these (65/293) were CRKP strains, and the proportion of CRKP-BSI in ICU was 59.62\% (31/52), equaling the levels observed in the epidemic regions. A number of KP-BSIs (114), obtained from January 1, 2014, to December 31, 2015, were further investigated. Skin and soft tissue infection source (odds ratio [OR] 26.63, 95\% confidence interval [Cl] 4.8-146.8) and ICU-acquired infection (OR 5.82, 95\% Cl 2.0-17.2) was shown to be powerful risk factors leading to the development of CRKP-BSI. The crude 28-day mortality rates of KP-BSI and CRKP-BSI patients were $22.8 \%$ and $33.3 \%$, respectively. Lung as the probable source of infection (OR 4.23,95\% Cl 1.0-17.3), and high Sequential Organ Failure Assessment (SOFA) score (OR 1.40, 95\% Cl 1.2-1.6) were strong prognostic factors determining crude 28-day KP-BSI mortality rates. PFGE analysis demonstrated that 10/11 random CRKP isolates in ICU belonged to the same clonal type.
\end{abstract}

Conclusions: During the study period, we observed a significant increase in the occurrence of CRKP infections among the identified KP-BSIs in our hospital and especially in ICU, and we demonstrated that carbapenem resistance is associated with the increased mortality of KP-BSI patients.

Keywords: Klebsiella pneumoniae, Bloodstream infection, Epidemiology, Carbapenem resistance, Mortality

\footnotetext{
*Correspondence: hongpingqu0412@hotmail.com; xiaoliwang0714@163.com

${ }^{\dagger}$ Equal contributors

${ }^{1}$ Department of Critical Care Medicine, Ruijin Hospital, Shanghai Jiao Tong

University School of Medicine, No.197 Ruijin ER Road, Shanghai 200025,

China

Full list of author information is available at the end of the article
} 


\section{Background}

Klebsiella pneumoniae (KP) is an important pathogen responsible for severe diseases such as septicemia, pneumonia, urinary tract infections, and soft tissue infections, and this pathogen is mainly associated with communityand hospital-acquired infections [1]. Nielsen et al. reported that $K$. pneumoniae is the second most common cause of gram-negative bloodstream infections (BSIs), after Escherichia coli, in adult population [2]. However, the incidence of KP-BSIs had exceeded the incidence of E. coli-caused BSIs in recent studies investigating intensive care unit (ICU) patients [3, 4]. High mortality rates, ranging from 20 to $40 \%$, were reported for patients with KP-BSI [5], but this incidence was reported to rise up to $67.6 \%$ for ICU patients [3].

Bacterial and host factors, as well as different treatment regimens, most likely influence the outcomes of patients with KP-BSI. Carbapenem-resistant $K$. pneumoniae (CRKP) infections may be associated with an increased rate of treatment failure and death [6], and the dramatic increase in the occurrence of CRKP worldwide aggravates this situation further [7]. Furthermore, older age, nosocomial infections, intensive care interventions, disease severity, and several comorbidities were reported to represent host factor contributing to the increased mortality rates of KP-BSI patients $[3,5,8,9]$. Additionally, $\beta$-lactams were the first-line antibiotics selected for the treatment of KPBSI patients, however, with the increase in the number of $K$. pneumoniae isolates producing extended-spectrum $\beta$ lactamase, the use of carbapenems increased as well. More importantly, CRKP isolates were shown to be resistant to a variety of antibiotics, and to have the ability to hydrolyze carbapenems, leaving few effective treatment options. The use of combination therapy and appropriate empirical treatment may provide useful prognostic information in these patients [10-12].

Although the high mortality rates have been extensively reported worldwide, to date, few studies have investigated the epidemiology of KP-BSIs in mainland China. The aim of this study was to describe the epidemiological, microbiological, and clinical characteristics of KP-BSIs in patients admitted to a teaching hospital in Shanghai, China, focusing on the risk factors related to carbapenem resistance and patient mortality.

\section{Methods}

\section{Study design and population}

Study design and all experimental procedures were approved by the Institutional Review Board of Ruijin Hospital, an 1800-bed tertiary care university teaching hospital in Shanghai, China. WHONET data provided by clinical microbiology laboratory were used to identify all KP-BSI patients between January 1, 2011, and December 31,2015 . Only the first KP-BSI reported for each patient was included in our analyses. These data were used to evaluate the rate of CRKP-BSIs.

We chose 114 incidences of KP-BSIs that occurred between January 1, 2014, and December 31, 2015 for further investigation. Clinical and microbiological characteristics and treatment outcomes were retrieved from the medical records by two experienced ICU medical doctors. The information about the patient demographics (age, gender), comorbidities (diabetes mellitus, chronic renal failure, chronic liver disease, biliary tract disease, congestive heart failure, chronic obstructive pulmonary disease, malignancy, and immunosuppression), probable source of infection, prior healthcare exposure conditions, such as major surgery in the past 30 days and the receipt of any antibiotic during $>48 \mathrm{~h}$ in the past 30 days, was collected. Additionally, the general state of patients on the onset of BSI was assessed, such as acute kidney injury, septic shock, use of mechanical ventilation, use of renal replacement therapy (RRT), and the removal of the probable infectious source. Comorbid conditions were also determined using the Charlson comorbidity index (CCI) as previously described [13], and the severity of illness during the onset of BSI was calculated by the Pitt bacteraemia score (PBS) and Sequential Organ Failure Assessment score (SOFA score) [14, 15]. Furthermore, the appropriate empirical antimicrobial therapies described by Zarkotou were considered as well [10]. We investigated attributable and crude in-hospital mortality rates and 28-day mortality rates of patients, in order to assess the treatment outcomes. Total length of hospital stay (LOS) and LOS after BSI were evaluated, while the primary outcome in this study was the crude 28-day mortality rate.

\section{Definitions}

BSI was defined according to the Centers for Disease Control and Prevention guidelines (available at: http:// www.cdc.gov/nhsn/pdfs/pscmanual/17pscnosinfdef_current.pdf). BSI onset was defined as the collection date of isolate. Nosocomial infection was defined as an infection that occurred more than $48 \mathrm{~h}$ after the admission of the patient to the hospital or an infection that existed in patients who had been admitted to other hospitals in 2 weeks before the current admission [16]. ICUacquired BSI was defined as the first positive blood culture identified more than 2 days after ICU admission (without a prior positive blood culture with the same pathogen for at least 30 days). The probable infectious source was determined on the basis of the microbiological results and the analysis by 2 physicians. When no source was identified, primary BSI was recorded. Appropriate empirical antimicrobial treatment refers to the administration of in vitro active antimicrobials against the study isolates, within $\leq 24 \mathrm{~h}$ from the BSI onset [10]. 
Septic shock was defined as sepsis associated with organ dysfunction and persistent hypotension despite volume replacement, and acute kidney injury (AKI) was defined using the established criteria [17, 18]. Crude mortality was defined as death occurring after the collection of the first blood culture positive for $K$. pneumoniae. Mortality attributable to BSI was defined by clinical evidence of active infection and positive cultures, or when death occurred as the result of organ failure that developed or deteriorated during the onset of infection [19].

\section{Microbiology}

Vitek 2 automated system (bioMérieux, Marcy l'Etoile, France) was used for isolate identification and antimicrobial susceptibility testing. Minimum inhibitory concentrations (MICs) were classified according to breakpoints established by the Clinical and Laboratory Standards Institute (CLSI2014) [20]. E. coli ATCC 25922 was used as a quality control reference strain. The genetic relatedness of CRKP strains obtained from ICU patients was determined by pulsed-field gel electrophoresis (PFGE) [21]. PFGE patterns were interpreted using the criteria proposed by Tenover et al. [22].

\section{Statistical analysis}

All results are expressed as the mean \pm standard deviation (SD) or median (interquartile range [IQR]) (continuous variables) or as percentages of the group from which they were derived (categorical variables). Student's $t$-test and Mann-Whitney $U$-test were used to compare normally and non-normally distributed continuous variables, respectively. Categorical variables were compared using the $\chi^{2}$ test or Fisher's test, as appropriate. Odds ratios (ORs) and $95 \%$ confidence intervals (CIs) were calculated for all emerging associations. Multivariate analysis was used to identify independent predictors by applying binary logistic regression. Variables with $p$-value $<0.05$ in univariate testing were incorporated into the model using a forward stepwise approach, and $p$-value $<0.05$ was set as the limit for the retention of the variables in the model. The goodness of fit for our logistic regression model was assessed with Hosmer-Lemeshow test. In multivariate analysis, comorbidities were substituted using the $\mathrm{CCI}$, and hospital events on the onset of BSI were replaced by PBS and SOFA score. Differences were considered to be significant for $p<0.05$. Data were analyzed with the IBM SPSS Statistics for Windows (version 19.0). All susceptibility data were analyzed using WHONET, version 5.6.

\section{Results}

Increased rates of CRKP-BSIs, especially among ICU patients

KP-BSIs were collected and analyzed using WHONET from January 1, 2011, to December 31, 2015 in our hospital. During this period, 293 episodes of KP-BSI were identified, and $22.18 \%(65 / 293)$ of these cases were CRKP isolates, while $47.7 \%(31 / 65)$ of the CRKP isolates were obtained from ICU. The annual rate of CRKP in clinical isolates in China, obtained from the CHINET surveillance of bacterial resistance [23], and the percent of CRKP-BSI determined in this study are presented in Fig. 1. The percentage of CRKP-BSIs in our hospital significantly increased from $14.5 \%$ in 2012 to $29.8 \%$ in 2015 , together with the percentage growth from $40 \%$ in

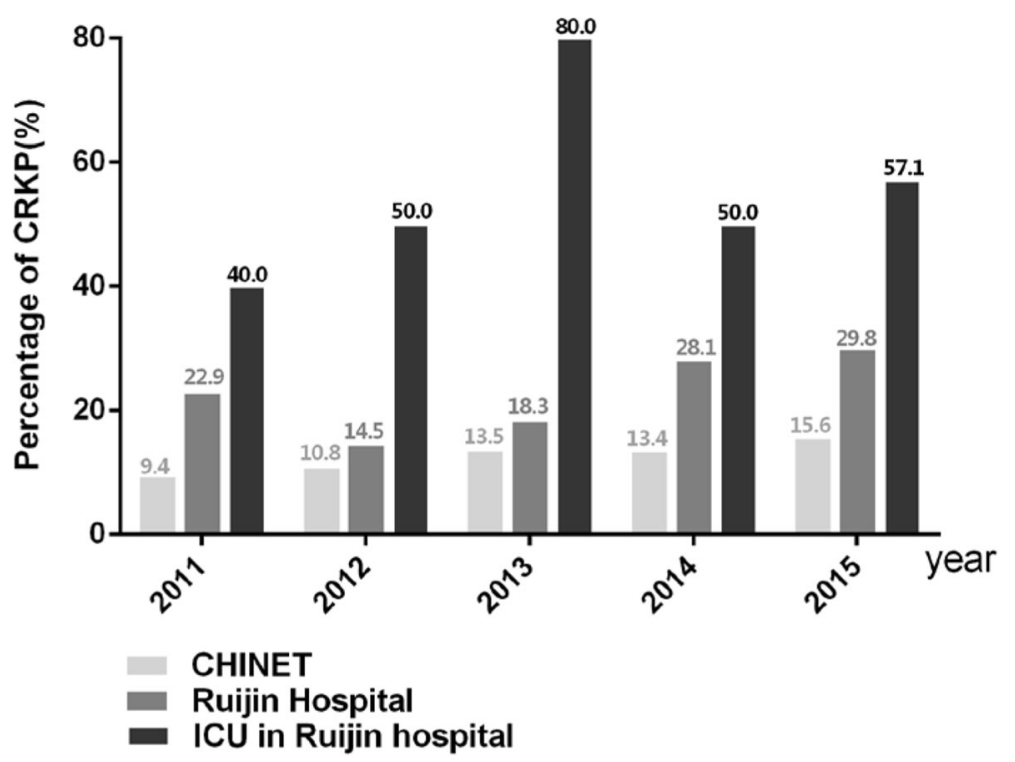

Fig. 1 CRKP among identified KP-BSIs from January 2011 to December 2015. Light grey bar, percentage of CRKP from CHINET; dark grey bar, percentage of CRKP-BSI in Ruijin Hospital from WHONET; black bar, percentage of CRKP-BSI in ICU of Ruijin Hospital from WHONET 
2011 to $57.1 \%$ in 2015 in the isolates from ICU patients, with the highest rate obtained in 2013, up to $80 \%$. A similar trend, with the rate of CRKP rising from 9.4\% in 2011 to $15.6 \%$ in 2015 was observed in data obtained from the CHINET surveillance program.

We demonstrated that the occurrence of CRKP strains causing KP-BSIs since 2011 is increasing, and the annual rates of these infections in the hospital included in this study are much higher than the national average. Additionally, the proportion of CRKP-BSIs in ICU patients was $59.62 \%(31 / 52)$, which is significantly higher than $14.11 \%(34 / 241)$ of the patients with these infections in non-ICU during this 5 -year period $(p<0.001)$.

\section{Risk factors associated with the development of CRKP- BSIs}

Because of the significant increase in the number of CRKP-BSI cases in our hospital since 2014 and the increased accuracy of the medical records, 114 incidences of KP-BSIs that occurred between January 1, 2014, and December 31, 2015, were further investigated. The mean age of these 114 patients was 56 years (median: 67, range: $12-90$ years), and $64.9 \%(74 / 114)$ of the patients were male. Most of the KP-BSIs (86.0\%) were nosocomial infections, and among them, 26 cases $(22.8 \%)$ were acquired at ICU. Most common infection sources were primary bloodstream infections (23.7\%), and intraabdominal (42.1\%) and lung (14.0\%) infections. The median CCI was 3 (IQR, 2-6) with the most frequent comorbidities being malignancy (51.8\%), biliary tract disease (30.7\%), and diabetes mellitus (26.3\%). The median PBS and SOFA score were 1 (IQR, 1-2.25) and 3 (IQR, 1-6), respectively.

To identify the clinical characteristics affecting the development of CRKP-BSIs, we compared the clinical characteristics of patients with CRKP-BSIs and CSKP-BSIs (Table 1). The factors determined to be significantly associated with CRKP-BSIs, using the univariate analysis, included nosocomial infection, ICU-acquired infection, probable infection source from skin and soft tissue, prior exposure to antimicrobial therapy in the past 30 days, and high PBS. In contrast, diabetes mellitus, malignancy, and high CCI were common in patients with CSKP-BSIs. Using multivariate analysis, skin and soft tissue infection source (OR 26.63, 95\%CI 4.8-146.8) and ICU-acquired infection (OR 5.82, 95\%CI 2.0-17.2) was determined to be independent factors for CRKP-BSI development.

\section{Comparison of antimicrobial therapies and treatment outcomes between CRKP-BSI and CSKP-BSI patients}

Antimicrobial therapies and outcomes of patients with KP-BSIs are presented in Table 2. Patients with CSKPBSI were more likely to receive appropriate empirical therapy than the patients with CRKP-BSI. Patients with
CRKP-BSI had longer total LOS compared with the patients with CSKP-BSI (median [IQR], 50 [28-83] vs. 24 [16.5-51], $p<0.001)$. The crude 28-day mortality and inhospital mortality of KP-BSI patients were $22.8 \%$ (26/ 114 ) and $26.3 \%(30 / 114)$, respectively. Crude in-hospital mortality was higher in patients with CRKP-BSIs than in those with CSKP-BSIs (42.4\% vs. $19.8 \%$, respectively, $p=$ $0.013)$, and this difference existed when crude 28-day mortality was compared $(33.3 \%$ vs. $18.5 \%$, respectively, $p=0.087$ ). Additionally, attributable 28-day mortality and in-hospital mortality of KP-BSI patients was $21.1 \%(24 / 114)$ and $24.6 \%$ (28/114), respectively. Similarly, in CRKP-BSI patients, the attributable 28-day mortality and in-hospital mortality were significantly higher than in CSKP-BSI patients $(33.3 \%$ vs. $16 \%$, $p=0.04 ; 42.4 \%$ vs. $24.6 \%, p=0.005$, respectively).

\section{Identification of clinical and microbiological characteristics associated with crude 28-day mortality}

A comparison of clinical and microbiological characteristics of the patients enrolled in this study is presented in Table 3. We aimed to identify the risk factors associated with crude 28-day mortality of KP-BSI patients. In the univariate analysis, ICU-acquired KP-BSI was determined to be a risk factor (OR 2.81, 95\% CI 1.1-7.3) and, when all comorbidities were analyzed, chronic liver disease (OR 3.71, 95\% CI 1.3-10.3) and congestive heart failure (OR 5.00, 95\% CI 1.2-20.2) were shown to be risk factors as well. Probable lung infection (OR 6.13, 95\% CI 2.0-18.7) was the source of these bloodstream infections more frequently in non-survivors than in survivors. Moreover, mortality rate was affected by the events on the onset of BSIs, such as acute kidney injury, septic shock, RRT use, and use of mechanical ventilation. Additionally, high SOFA score (median [IQR], 9 [4-13.5] vs. $2[1-4], p<0.001)$ and high PBS (median [IQR], 5 [2-7] vs. $1[1,2], p<0.001)$ were associated the crude 28-day mortality in univariate analyses. There was no statistical difference in hospital events prior to the onset of BSI between survivors and non-survivors. Obtained using multivariate logistic regression analysis, significant risk factors for crude 28-day mortality were lungs as probable source of infection (OR 4.23, 95\% CI 1.0-17.3), and high SOFA score (OR 1.40, 95\% CI 1.2-1.6), after adjusting for other confounding variables.

\section{PFGE analysis of random CRKP ICU isolates}

To further determine the genetic relatedness of CRKP strains in ICU, we randomly selected 11 CRKP ICU isolates for PFGE analysis. The obtained results indicated that these isolates belong to two different clonal types (Fig. 2). The predominant clonal type was shown to be A- 
Table 1 Comparison of clinical characteristics between CRKP-BSI and CSKP-BSI patients

\begin{tabular}{|c|c|c|c|c|c|c|c|}
\hline \multirow[t]{2}{*}{ Variable } & \multirow[t]{2}{*}{ Total $(n=114) \mathrm{n}(\%)$} & \multirow[t]{2}{*}{ CRKP $(n=33) n(\%)$} & \multirow[t]{2}{*}{$\operatorname{CSKP}(n=81) n(\%)$} & \multicolumn{2}{|c|}{ Univariate analysis } & \multicolumn{2}{|c|}{ Multivariate analysis } \\
\hline & & & & $\mathrm{OR}(95 \% \mathrm{Cl})$ & $p$-value & $\mathrm{OR}(95 \% \mathrm{Cl})$ & $p$-value \\
\hline \multicolumn{8}{|l|}{ General variables } \\
\hline Male sex & $74(64.9)$ & $22(66.7)$ & $52(64.2)$ & $1.12(0.5-2.6)$ & 0.802 & & \\
\hline Age,years, mean \pm SD & $56.37 \pm 16.36$ & $51.70 \pm 16.26$ & $58.27 \pm 16.11$ & NA & 0.051 & & \\
\hline Poly-microbial BSI & $11(9.6)$ & $6(18.2)$ & $5(6.2)$ & $3.38(1.0-12.0)$ & 0.076 & & \\
\hline Nosocomial infection & 98(86.0) & $33(100)$ & $65(80.2)$ & NA & 0.005 & - & - \\
\hline ICU acquired infection & $26(22.8)$ & $14(42.4)$ & $12(14.8)$ & $4.24(1.7-10.7)$ & 0.001 & $5.82(2.0-17.2)$ & 0.002 \\
\hline \multicolumn{8}{|l|}{ Comorbidities } \\
\hline Diabetes mellitus & $30(26.3)$ & $3(9.1)$ & $27(33.3)$ & $0.20(0.1-0.7)$ & 0.008 & & \\
\hline Chronic renal failure & $8(7.0)$ & $3(9.1)$ & $5(6.2)$ & $1.52(0.3-6.8)$ & 0.689 & & \\
\hline Chronic liver disease & $20(17.5)$ & $5(15.2)$ & $15(18.5)$ & $0.79(0.3-2.4)$ & 0.668 & & \\
\hline Liver cirrhosis & 11(9.6) & $2(6.1)$ & $9(11.1)$ & $0.52(0.1-2.5)$ & 0.506 & & \\
\hline Biliary tract disease & $35(30.7)$ & $9(27.3)$ & $26(32.1)$ & $0.79(0.3-1.9)$ & 0.612 & & \\
\hline Congestive heart failure & $9(7.9)$ & $4(12.1)$ & $5(6.2)$ & $2.10(0.5-8.4)$ & 0.280 & & \\
\hline $\begin{array}{l}\text { Chronic obstructive } \\
\text { pulmonary disease }\end{array}$ & $3(2.6)$ & $1(3.0)$ & $2(2.5)$ & $1.23(0.1-14.1)$ & 1 & & \\
\hline Malignancy & 59(51.8) & $12(36.4)$ & $47(58.0)$ & $0.41(0.2-1.0)$ & 0.036 & & \\
\hline Immunosuppression & $26(22.8)$ & $10(30.3)$ & $16(19.8)$ & $1.77(0.7-4.4)$ & 0.223 & & \\
\hline $\mathrm{CCl}$,median (IQR) & $3(2-6)$ & $3(0-4)$ & $4(2-6)$ & NA & 0.005 & - & - \\
\hline \multicolumn{8}{|l|}{ Probable source of infection } \\
\hline Lung & $16(14.0)$ & $5(15.2)$ & $11(13.6)$ & $1.14(0.4-3.6)$ & 0.776 & & \\
\hline Urinary & $1(0.9)$ & $0(0)$ & $1(0.9)$ & NA & 1 & & \\
\hline Intra-abdominal & $48(42.1)$ & $12(36.4)$ & $36(44.4)$ & $0.71(0.3-1.6)$ & 0.428 & & \\
\hline Liver abscess & $6(5.3)$ & $1(3.0)$ & $5(6.2)$ & $0.48(0.1-4.2)$ & 0.671 & & \\
\hline Skin and soft tissue & $12(10.5)$ & $10(30.3)$ & $2(2.5)$ & $17.17(3.5-84.0)$ & $<0.001$ & $26.63(4.8-146.8)$ & $<0.001$ \\
\hline Catheter & 10(8.8) & $5(15.2)$ & $5(6.2)$ & $2.71(0.7-10.1)$ & 0.150 & & \\
\hline Primary & $27(23.7)$ & $5(15.2)$ & $22(27.2)$ & $0.48(0.2-1.4)$ & 0.171 & & \\
\hline
\end{tabular}


Table 1 Comparison of clinical characteristics between CRKP-BSI and CSKP-BSI patients (Continued)

\begin{tabular}{|c|c|c|c|c|c|c|c|}
\hline \multicolumn{8}{|l|}{$\begin{array}{l}\text { Hospital events prior to } \\
\text { onset of BSI }\end{array}$} \\
\hline $\begin{array}{l}\text { Exposure to antimicrobial } \\
\text { therapy }{ }^{a}\end{array}$ & $74(64.9)$ & 30(90.9) & $44(54.3)$ & $8.41(2.4-29.8)$ & $<0.001$ & $4.04(1.0-16.5)$ & 0.052 \\
\hline Surgery ${ }^{a}$ & $62(54.4)$ & $21(63.6)$ & $41(50.6)$ & $1.71(0.7-3.9)$ & 0.206 & & \\
\hline \multicolumn{8}{|l|}{ Events on the onset of BSI } \\
\hline SOFA score,median (IQR) & $3(1-6)$ & $4(1-7)$ & $3(1-5)$ & NA & 0.098 & & \\
\hline PBS,median (IQR) & $1(1-2.25)$ & $2(1-5)$ & $1(0-2)$ & NA & $<0.001$ & - & - \\
\hline
\end{tabular}

in the past 30 days prior to onset of BSI $S D$, standard deviation, IQR interquartile range, $O R$ odds ratio, CI confidence interval, ICU intensive care unit, BSI bloodstream infection, CRKP carbapenem-resistant Klebsiella pne
Klebsiella pneumoniae, LOS length of stay, CCI Charlson comorbidity index, PBS Pitt bacteraemia score, SOFA score sequential organ failure assessment score, NA non-applicable 
Table 2 Comparison of antimicrobial therapies and treatment outcomes between CRKP-BSI and CSKP-BSI patients

\begin{tabular}{|c|c|c|c|c|}
\hline Treatment and outcomes & Total $(n=114)$ & $\operatorname{CRKP}(n=33)$ & $\operatorname{CSKP}(n=81)$ & $p$-value \\
\hline Appropriate empirical antimicrobial therapy & $78(68.4)$ & $9(27.3)$ & $69(85.2)$ & $<0.001$ \\
\hline LOS after BSI onset,Days, median (IQR) & 18.5(9-31) & $24(9.5-51)$ & $15(8.5-28)$ & 0.066 \\
\hline Total LOS,days, median (IQR) & $31.5(19.75-62.75)$ & $50(28-83)$ & $24(16.5-51)$ & 0.001 \\
\hline \multicolumn{5}{|l|}{ Mortality rate } \\
\hline Crude 28-day mortality n(\%) & $26(22.8)$ & $11(33.3)$ & 15(18.5) & 0.087 \\
\hline Crude in-hospital mortality n(\%) & $30(26.3)$ & 14(42.4) & 16(19.8) & 0.013 \\
\hline Attributable 28-day mortality n(\%) & 24(21.1) & 11(33.3) & 13(16.0) & 0.04 \\
\hline Attributable in-hospital mortality n(\%) & $28(24.6)$ & 14(42.4) & $14(24.6)$ & 0.005 \\
\hline
\end{tabular}

IQR interquartile range, $B S I$ bloodstream infection, CRKP carbapenem-resistant Klebsiella pneumoniae, CSKP carbapenem-sensitive Klebsiella pneumoniae, LOS length of stay

type (10 isolates [90.9\%], including A1-subtype [ $n=9]$ and A2-subtype $[n=1])$.

\section{Discussion}

The increase in the morbidity and mortality of KP-BSIs has attracted a lot of attention recently, together with the worldwide spread of hypervirulent and multidrugresistant $K$. pneumoniae strains. To the best of our knowledge, this is the largest 5-year epidemiological study of KP-BSIs in mainland China to date. In China, CHINET data showed that the rate of carbapenem resistance of $K$. pneumoniae isolates rose slowly from $3.0 \%$ in 2005 to $4.9 \%$ in 2009, but a rapid increase in the number of CRKP isolates has been reported since 2010, with the current national average as high as $15.6 \%$ [23]. Over the past 10 years, the rate of CRKP has also increased dramatically worldwide [7], and in the areas of high CRKP endemicity, such as the United States, Israel, Italy, and Greece, the percentage of CRKP-BSI was shown to be $18-68 \%[19,24-26]$. In agreement with these results, we observed an increase in CRKP-BSI rates in our hospital after 2011, and the obtained data shows that these rates are significantly higher than the average rates in China, especially among ICU patients, although the rate of CRKP-BSIs in non-ICU patients increased, rising up to $20 \%$ since 2014. Noteworthy, the incidence of CRKP-BSIs in our ICU was higher than $50 \%$, which is similar to the rates observed in the endemic areas [25,27], however, it may be possible that the analyses focused on the patient vulnerability and disease severity, and several patients were transferred from the endemic CRKP regions of China, such as Zhejiang and Jiangxi provinces [28].

Clinical characteristics associated with CRKP-BSI patients in our study were similar to those reported by other studies, with the exception of probable skin and soft tissue infection source $[19,29,30]$. Jolie et al. reported that patients with $\geq 20 \%$ total body surface area burns show a higher risk of bacteremia [31], and Bang et $a l$. found that the patients with extensive flame burns are prone to septicemia [32]. In our study,patients with
KP-BSIs, with the infection source from skin and soft tissue, were accepted to the burn center of the hospital, and all suffered from flame injuries. In 10 out of these 12 patients, burns were found on over $30 \%$ of total body surface area, and they underwent surgeries, usually comprised of debridement and grafting, and most of them received antibiotic therapies containing third or fourth generation cephalosporins or carbapenems. A previous 9-year-long study confirmed that CRKP incidences increase significantly in severe burn patients with late BSIs [33]. Additionally, Cen et al. determined that the rate of CRKP strains has increased in recent years, although Pseudomonas aeruginosa and Acinetobacter baumannii were more frequently detected gram-negative bacteria in a Chinese burn ward [34]. This suggests that it is vital to protect the integrity of skin and mucosal barriers in the critically ill patients or the patients undergoing invasive procedures, in order to prevent the development of antibiotic-resistant bacterial infections. All necessary contact precautions should be employed by hospital staff caring for these high-risk patients.

Interestingly, CSKP-BSIs were especially common among the patients with diabetes mellitus, which may be related to hypermucoviscous K. pneumoniae (HVKP). Diabetes mellitus has been considered a significant risk factor for HVKP, prevalent in the Asian Pacific Rim since the 1980s [35]. Our unpublished data showed a high rate of HVKP isolates in our hospital, and the overwhelming majority of them were CSKP.

The crude 28-day mortality in KP-BSI patients was shown to be $22.8 \%$, which increased to $33.3 \%$ in CRKPBSI patients, similar to the rates obtained in both Chinese and foreign studies [12, 25, 29, 36-38]. Furthermore, the mortality of CRKP-BSI patients was shown to be higher than that of CSKP-BSI patients, which may be a consequence of the infection with ST11 clone, which is a dominant CRKP strain in China [28] with an increased pathogenic potential and resistance to serum killing $[39,40]$. Additionally, due to the limited CRKP treatment options, inappropriate empirical therapy may 
Table 3 Identification of clinical and microbiological characteristics associated with crude 28-day mortality

\begin{tabular}{|c|c|c|c|c|c|c|c|}
\hline \multirow[t]{2}{*}{ Variable } & \multirow[t]{2}{*}{ Total $(n=114) n(\%)$} & \multirow[t]{2}{*}{ Death $(n=26) \mathrm{n}(\%)$} & \multirow[t]{2}{*}{ Survivors $(n=88) \mathrm{n}(\%)$} & \multicolumn{2}{|c|}{ Univariate analysis } & \multicolumn{2}{|c|}{ Multivariate analysis } \\
\hline & & & & $\mathrm{OR}(95 \% \mathrm{Cl})$ & $p$-value & $\mathrm{OR}(95 \% \mathrm{Cl})$ & $p$-value \\
\hline \multicolumn{8}{|l|}{ General variables } \\
\hline Male sex & $74(64.9)$ & $20(76.9)$ & $54(61.4)$ & $2.10(0.8-5.8)$ & 0.144 & & \\
\hline Age,years, mean \pm SD & $56.37 \pm 16.36$ & $55.54 \pm 16.10$ & $56.61 \pm 16.52$ & NA & 0.770 & & \\
\hline Poly-microbial BSI & $11(9.6)$ & $3(11.5)$ & $8(9.1)$ & $1.30(0.3-5.3)$ & 0.711 & & \\
\hline Nosocomial infection & $98(86.0)$ & $23(88.5)$ & $75(85.2)$ & $1.33(0.3-5.1)$ & 1 & & \\
\hline ICU acquired infection & $26(22.8)$ & $10(38.5)$ & $16(18.2)$ & $2.81(1.1-7.3)$ & 0.03 & - & - \\
\hline \multicolumn{8}{|l|}{ Comorbidities } \\
\hline Diabetes mellitus & $30(26.3)$ & $4(15.4)$ & $26(29.5)$ & $0.43(0.1-1.4)$ & 0.150 & & \\
\hline Chronic renal failure & $8(7.0)$ & $1(3.8)$ & $7(8.0)$ & $0.463(0.05-3.9)$ & 0.680 & & \\
\hline Chronic liver disease & $20(17.5)$ & $9(34.6)$ & $11(12.5)$ & $3.71(1.3-10.3)$ & 0.017 & & \\
\hline Liver cirrhosis & $11(9.6)$ & $5(19.2)$ & $6(6.8)$ & $3.25(0.9-11.7)$ & 0.122 & & \\
\hline Biliary tract disease & $35(30.7)$ & $8(30.8)$ & $27(30.7)$ & $1.00(0.4-2.6)$ & 1 & & \\
\hline Congestive heart failure & $9(7.9)$ & $5(19.2)$ & $4(4.5)$ & $5.00(1.2-20.2)$ & 0.028 & & \\
\hline $\begin{array}{l}\text { Chronic obstructive } \\
\text { pulmonary disease }\end{array}$ & $3(2.6)$ & $0(0)$ & $3(3.4)$ & NA & 1 & & \\
\hline Malignancy & $59(51.8)$ & $11(42.3)$ & $48(54.5)$ & $0.61(0.3-1.5)$ & 0.273 & & \\
\hline Immunosuppression & $26(22.8)$ & $6(23.1)$ & $20(22.7)$ & $1.02(0.4-2.9)$ & 0.97 & & \\
\hline $\begin{array}{l}\text { Charlson comorbidity } \\
\text { index, median (IQR) }\end{array}$ & $3(2-6)$ & $3(2-6.75)$ & $3(1.25-6)$ & NA & 0.576 & & \\
\hline \multicolumn{8}{|l|}{ Probable source of infection } \\
\hline Lung & $16(14.0)$ & $9(34.6)$ & $7(8.0)$ & $6.13(2.0-18.7)$ & 0.002 & $4.23(1.0-17.3)$ & 0.045 \\
\hline Intra-abdominal & $48(42.1)$ & $9(34.6)$ & $39(44.3)$ & $0.67(0.3-1.7)$ & 0.379 & & \\
\hline Skin and soft tissue & $12(10.5)$ & $4(15.4)$ & $8(9.1)$ & $1.82(0.5-6.6)$ & 0.465 & & \\
\hline Catheter-related & 10(8.8) & $2(7.7)$ & $8(9.1)$ & $0.83(0.2-4.2)$ & 1 & & \\
\hline \multicolumn{8}{|l|}{$\begin{array}{l}\text { Hospital events prior to } \\
\text { onset of BSI }\end{array}$} \\
\hline $\begin{array}{l}\text { Exposure to antimicrobial } \\
\text { therapy }{ }^{a}\end{array}$ & $74(64.9)$ & $20(76.9)$ & $54(61.4)$ & $2.10(0.8-5.8)$ & 0.144 & & \\
\hline Surgery ${ }^{a}$ & $62(54.4)$ & $15(57.7)$ & $47(53.4)$ & $1.19(0.5-2.9)$ & 0.700 & & \\
\hline
\end{tabular}


Table 3 Identification of clinical and microbiological characteristics associated with crude 28-day mortality (Continued)

\begin{tabular}{|c|c|c|c|c|c|c|c|}
\hline \multicolumn{8}{|l|}{ Events on the onset of BSI } \\
\hline Acute kidney injury & $16(14.0)$ & $12(46.2)$ & $4(4.5)$ & $18.00(5.1-63.8)$ & $<0.001$ & & \\
\hline $\begin{array}{l}\text { Use of renal replacement } \\
\text { therapy }\end{array}$ & $8(7.0)$ & $5(19.2)$ & $3(3.4)$ & $6.75(1.5-30.5)$ & 0.015 & & \\
\hline Septic shock & $28(24.6)$ & $16(61.5)$ & 12(13.6) & $10.13(3.7-27.5)$ & $<0.001$ & & \\
\hline $\begin{array}{l}\text { Use of mechanical } \\
\text { ventilation }\end{array}$ & $24(21.1)$ & $15(57.7)$ & $9(10.2)$ & 11.97(4.2-33.9) & $<0.001$ & & \\
\hline $\begin{array}{l}\text { Removal of the infectious } \\
\text { source }\end{array}$ & $33(28.9)$ & $6(23.1)$ & 27(30.7) & $0.68(0.2-1.9)$ & 0.453 & & \\
\hline SOFA score, median (IQR) & $3(1-6)$ & $9(4-13.5)$ & $2(1-4)$ & NA & $<0.001$ & $1.40(1.2-1.6)$ & $<0.001$ \\
\hline $\begin{array}{l}\text { Pitt bacteraemia score, } \\
\text { median (IQR) }\end{array}$ & $1(1-2.25)$ & $5(2-7)$ & $1(1-2)$ & NA & $<0.001$ & - & - \\
\hline CRKP & $33(28.9)$ & $11(42.3)$ & $22(25.0)$ & $2.20(0.9-5.5)$ & 0.087 & & \\
\hline Appropriate empirical therapy & $78(68.4)$ & $14(53.8)$ & $64(72.7)$ & $0.44(0.2-1.1)$ & 0.069 & & \\
\hline
\end{tabular}

${ }^{\mathrm{in}}$ the past 30 days prior to onset of BSI
$S D$ standard deviation, IQR interquartile range, OR odds ratio, CI confidence interval, ICU intensive care unit, BSI bloodstream infection, CRKP carbapenem-resistant Klebsiella pneumoniae, CSKP carbapenem-sensitive Klebsiella pneumoniae, LOS length of stay, CCI Charlson comorbidity index, PBS Pitt bacteraemia score, SOFA score sequential organ failure assessment score 


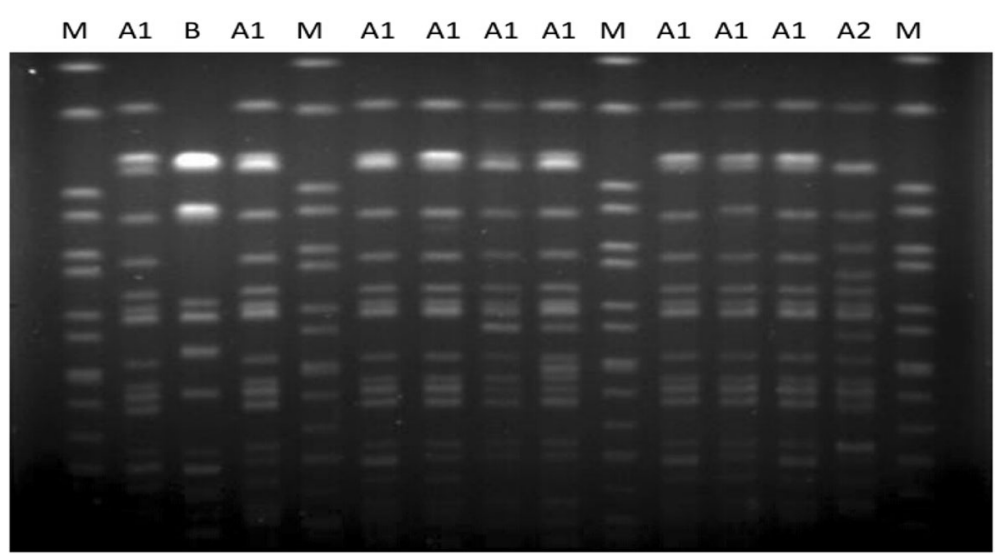

Fig. 2 PFGE analysis of random CRKP ICU isolates. Genomic DNA was digested using Xbal enzyme and subjected to PFGE. PFGE patterns were interpreted by using the criteria proposed by Tenover et al. [24]. Lane M, a Salmonella serotype Braenderup strain (H9812) was used to normalize migration variation occurring across the gel and to accurately determine sample band sizes

contribute to their deleterious outcomes [4, 19]. According to the recent clinical observations, the treatment of CRKP infections by a combination therapy may result in the reduced mortality compared with monotherapy $[11,12]$, but the treatment should be further optimized.

To further investigate the risk factors associated with crude 28-day mortality, we evaluated the influence of patient characteristics, $K$. pneumoniae isolates, and the therapeutic interventions on KP-BSIs. After adjusting for multiple confounders, several parameters, such as probable source of infection from lung and high SOFA score, were associated with a higher crude 28-day mortality, as previously described [9, 41, 42]. For patients with SOFA scores above 7 at the onset of bacteremia, the 14-day mortality rate was shown to increase [43]. Here, a median SOFA score at the onset of CRKP-BSI was 9. Therefore, SOFA score determined at the onset of BSI may capture the risk associated with the severity of BSI or the clinical impact of any other condition. It may help physicians identify the patients with BSIs who have a higher risk of in-hospital death. No significant difference was observed between the inappropriate empirical therapy and mortality, however, carbapenem resistance was not found to be an independent predictor of mortality, as previously reported [44], but such trend was observed, which may be explained by a relatively small sample population included in this study.

Increasing antibiotic resistance in ICUs represents a major clinical concern, and we showed here that almost half of all CRKP isolates were obtained from ICU patients, while further analysis showed that CRKP-BSIs are associated with ICU acquired infection, which may lead to higher mortality rates (38.5\%). Different risk factors for BSI development may affect patients in ICUs, including the higher disease severity, disruption of anatomical barriers, and impaired immunological response [45], which may lead to the increase in the length of hospital stay, worse outcomes, and the difficulties in the treatment [46, 47]. Furthermore, we analyzed the genetic relatedness of CRKP strains isolated from ICU patients, showing that the randomly isolated CRKP strains are almost identical, which may result from a nosocomial clonal expansion. It is worth noting that the highest rate of CRKP-BSIs in ICU rose up to $80 \%$ in 2013, but later, this percentage apparently decreased, most likely because of an integrated stewardship prevention study that was conducted in our ICU from February 1, 2014, to August 31, 2015. In this period, infection-control measures, such as active surveillance culture, isolation precautions, enhanced contact precautions, disinfection, and sterilization were applied. Therefore, prompt and appropriate infection control should be implemented upon the admission of high-risk patients, especially in ICU.

Our study has several limitations. The extended spectrum $ß$ lactamase-producing $K$. pneumoniae strains represent a major issue when analyzing multidrugresistant $K$. pneumoniae, however, we have not discussed this in our study. Clinical data were obtained retrospectively from medical records, and therefore, some differences in physician practices or accuracy of information may exist. Additionally, the data for patients who may have had significant BSI symptoms such as septic shock and hyperpyrexia, but were not tested due to the patient refusal or because their blood culture was negative, were not included. Finally, this was a single-center study, including 114 patients with detailed clinical analysis, and further multi-centric, prospective studies are needed to confirm our findings and to assist physicians in adopting more effective approaches for KP-BSI treatment. 


\section{Conclusions}

A significant increase in the occurrence of CRKP among the identified KP-BSIs in our hospital was observed, especially in ICU since 2011. We demonstrated that skin and soft tissue infection source and ICU-acquired infection represent strong risk factors for CRKP-BSI development. Probable lung infection source and high SOFA score were independent predictors for crude 28-day mortality of KP-BSI patients. Taken together, our results clearly demonstrated that CRKP-BSIs are associated with high morbidity and mortality, and clone spread may contribute to the nosocomial dissemination of CRKP, requiring the implementation of appropriate infection control, especially in ICU.

\section{Abbreviations \\ BSI: Bloodstream infection; CCl: Charlson comorbidity index; Cl: Confidence interval; CRKP: Carbapenem-resistant Klebsiella pneumoniae: \\ CSKP: Carbapenem-sensitive Klebsiella pneumoniae; HVKP: Hypermucoviscous Klebsiella pneumoniae; ICU: Intensive care unit; IQR: Interquartile range; KP: Klebsiella pneumoniae; LOS: Length of stay; NA: Non-applicable; OR: Odds ratio; PBS: Pitt bacteraemia score; PFGE: Pulsed-field gel electrophoresis; SD: Standard deviation; SOFA score: Sequential organ failure assessment score;}

\section{Acknowledgements}

The authors are grateful to Prof. Xiaokui Guo (Department of Microbiology and Parasitology, Shanghai Jiaotong University School of Medicine) for technical assistance with PFGE.

\section{Funding}

This work was financially supported by Shanghai Association for Science and Technology (grant numbers: 124119a6100, 134119b0200).

\section{Availability of data and material}

The raw data can be made available to the interested researchers by the authors of this article if requested.

\section{Authors' contributions \\ $L T, R T, X W$, and HQ designed the study, collected and analyzed the epidemiological data from the patient records, and wrote the manuscript. YC collected the isolates and performed PFGE. JS provided the microbiological data, while $J \mathrm{~L}$ monitored the study. All authors read and approved the final manuscript.}

\section{Competing interests}

The authors declare that they have no competing interests.

\section{Consent for publication}

Not applicable.

\section{Ethics approval and consent to participate}

Ethical approval was obtained from the Institutional Review Board of Ruijin Hospital, and the need for individual patient consent was waived (reference number: 201329).

\section{Author details}

'Department of Critical Care Medicine, Ruijin Hospital, Shanghai Jiao Tong University School of Medicine, No.197 Ruijin ER Road, Shanghai 200025, China. ${ }^{2}$ Department of Clinical Microbiology, Ruijin Hospital, Shanghai Jiao Tong University School of Medicine, No.197 Ruijin ER Road, Shanghai 200025, China.

Received: 17 August 2016 Accepted: 26 October 2016 Published online: 17 November 2016

\section{References}

1. Podschun R, Ullmann U. Klebsiella spp. as nosocomial pathogens: epidemiology, taxonomy, typing methods, and pathogenicity factors. Clin Microbiol Rev. 1998:11(4):589-603.

2. Nielsen SL, Pedersen C, Jensen TG, Gradel KO, Kolmos HJ, Lassen AT. Decreasing incidence rates of bacteremia: a 9-year population-based study. J Infect. 2014;69(1):51-9.

3. Delle Rose D, Sordillo P, Gini S, Cerva C, Boros S, Rezza G, Meledandri M, Gallo MT, Prignano G, Caccese R, et al. Microbiologic characteristics and predictors of mortality in bloodstream infections in intensive care unit patients: A 1-year, large, prospective surveillance study in 5 Italian hospitals. Am J Infect Control. 2015;43(11):1178-83.

4. Tabah A, Koulenti D, Laupland K, Misset B, Valles J, Bruzzi de Carvalho F, Paiva JA, Cakar N, Ma X, Eggimann P, et al. Characteristics and determinants of outcome of hospital-acquired bloodstream infections in intensive care units: the EUROBACT International Cohort Study. Intensive Care Med. 2012; 38(12):1930-45.

5. Meatherall BL, Gregson D, Ross T, Pitout JD, Laupland KB. Incidence, risk factors, and outcomes of Klebsiella pneumoniae bacteremia. Am J Med. 2009;122(9):866-73.

6. Patel G, Huprikar S, Factor SH, Jenkins SG, Calfee DP. Outcomes of carbapenem-resistant Klebsiella pneumoniae infection and the impact of antimicrobial and adjunctive therapies. Infect Control Hosp Epidemiol. 2008; 29(12):1099-106.

7. Gupta N, Limbago BM, Patel JB, Kallen AJ. Carbapenem-resistant Enterobacteriaceae: epidemiology and prevention. Clin Infect Dis. 2011; 53(1):60-7.

8. Al-Hasan MN, Lahr BD, Eckel-Passow JE, Baddour LM. Epidemiology and outcome of Klebsiella species bloodstream infection: a population-based study. Mayo Clin Proc. 2010;85(2):139-44.

9. Chetcuti Zammit S, Azzopardi N, Sant J. Mortality risk score for Klebsiella pneumoniae bacteraemia. Eur J Intern Med. 2014;25(6):571-6.

10. Zarkotou O, Pournaras S, Tselioti P, Dragoumanos V, Pitiriga V, Ranellou K, Prekates A, Themeli-Digalaki K, Tsakris A. Predictors of mortality in patients with bloodstream infections caused by KPC-producing Klebsiella pneumoniae and impact of appropriate antimicrobial treatment. Clin Microbiol Infect. 2011;17(12):1798-803.

11. Tumbarello M, Viale $P$, Viscoli $C$, Trecarichi EM, Tumietto F, Marchese A, Spanu T, Ambretti S, Ginocchio F, Cristini F, et al. Predictors of mortality in bloodstream infections caused by Klebsiella pneumoniae carbapenemaseproducing K. pneumoniae: importance of combination therapy. Clin Infect Dis. 2012;55(7):943-50.

12. Gomez-Simmonds A, Nelson B, Eiras DP, Loo A, Jenkins SG, Whittier S, Calfee DP, Satlin MJ, Kubin CJ, Furuya EY. Combination Regimens for Treatment of Carbapenem-Resistant Klebsiella pneumoniae Bloodstream Infections. Antimicrob Agents Chemother. 2016;60(6):3601-7.

13. Charlson ME, Pompei $P$, Ales KL, MacKenzie CR. A new method of classifying prognostic comorbidity in longitudinal studies: development and validation. J Chronic Dis. 1987:40(5):373-83.

14. Korvick JA, Bryan CS, Farber B, Beam Jr TR, Schenfeld L, Muder RR, Weinbaum D, Lumish R, Gerding DN, Wagener MM, et al. Prospective observational study of Klebsiella bacteremia in 230 patients: outcome for antibiotic combinations versus monotherapy. Antimicrob Agents Chemother. 1992;36(12):2639-44.

15. Vincent JL, de Mendonca A, Cantraine F, Moreno R, Takala J, Suter PM, Sprung $\mathrm{CL}$, Colardyn F, Blecher S. Use of the SOFA score to assess the incidence of organ dysfunction/failure in intensive care units: results of a multicenter, prospective study. Working group on "sepsis-related problems" of the European Society of Intensive Care Medicine. Crit Care Med. 1998; 26(11):1793-800.

16. Garner JS, Jarvis WR, Emori TG, Horan TC, Hughes JM. CDC definitions for nosocomial infections, 1988. Am J Infect Control. 1988;16(3):128-40.

17. Dellinger RP, Levy MM, Rhodes A, Annane D, Gerlach H, Opal SM, Sevransky JE, Sprung CL, Douglas IS, Jaeschke R, et al. Surviving sepsis campaign: international guidelines for management of severe sepsis and septic shock: 2012. Crit Care Med. 2013;41(2):580-637.

18. Kellum JA, Lameire N. Diagnosis, evaluation, and management of acute kidney injury: a KDIGO summary (Part 1). Crit Care. 2013;17(1):204.

19. Ben-David D, Kordevani R, Keller N, Tal I, Marzel A, Gal-Mor O, Maor Y, Rahav G. Outcome of carbapenem resistant Klebsiella pneumoniae bloodstream infections. Clin Microbiol Infect. 2012;18(1):54-60. 
20. Clinical and Laboratory Standards Institute. Performance standards for antimicrobial susceptibility testing-twenty fourth edition: approved standard M100eS24. Wayne: CLSI; 2014.

21. Ribot EM, Fair MA, Gautom R, Cameron DN, Hunter SB, Swaminathan B, Barrett TJ. Standardization of pulsed-field gel electrophoresis protocols for the subtyping of Escherichia coli 0157:H7, Salmonella, and Shigella for PulseNet. Foodborne Pathog Dis. 2006;3(1):59-67.

22. Tenover FC, Arbeit RD, Goering RV, Mickelsen PA, Murray BE, Persing DH, Swaminathan B. Interpreting chromosomal DNA restriction patterns produced by pulsed-field gel electrophoresis: criteria for bacterial strain typing. J Clin Microbiol. 1995;33(9):2233-9.

23. Hu FP, Guo Y, Zhu DM, Wang F, Jiang XF, Xu YC, Zhang XJ, Zhang CX, Ji $P$, $X i e Y$, et al. Resistance trends among clinical isolates in China reported from CHINET surveillance of bacterial resistance, 2005-2014. Clin Microbiol Infect. 2016;22 Suppl 1:S9-14.

24. Gomez-Simmonds A, Greenman M, Sullivan SB, Tanner JP, Sowash MG, Whittier S, Uhlemann AC. Population Structure of Klebsiella pneumoniae Causing Bloodstream Infections at a New York City Tertiary Care Hospital: Diversification of Multidrug-Resistant Isolates. J Clin Microbiol. 2015;53(7):2060-7.

25. Alicino C, Giacobbe DR, Orsi A, Tassinari F, Trucchi C, Sarteschi G, Copello F, Del Bono V, Viscoli C, Icardi G. Trends in the annual incidence of carbapenem-resistant Klebsiella pneumoniae bloodstream infections: a 8year retrospective study in a large teaching hospital in northern Italy. BMC Infect Dis. 2015;15:415.

26. Daikos GL, Tsaousi S, Tzouvelekis LS, Anyfantis I, Psichogiou M, Argyropoulou A, Stefanou I, Sypsa V, Miriagou V, Nepka M, et al. Carbapenemase-producing Klebsiella pneumoniae bloodstream infections: lowering mortality by antibiotic combination schemes and the role of carbapenems. Antimicrob Agents Chemother. 2014;58(4):2322-8.

27. Nordmann P, Cuzon G, Naas T. The real threat of Klebsiella pneumoniae carbapenemase-producing bacteria. Lancet Infect Dis. 2009;9(4):228-36.

28. Qi Y, Wei Z, Ji S, Du X, Shen P, Yu Y. ST11, the dominant clone of KPC producing Klebsiella pneumoniae in China. J Antimicrob Chemother. 2011; 66(2):307-12.

29. Girometti N, Lewis RE, Giannella M, Ambretti S, Bartoletti M, Tedeschi S, Tumietto F, Cristini F, Trapani F, Gaibani P, et al. Klebsiella pneumoniae bloodstream infection: epidemiology and impact of inappropriate empirical therapy. Medicine. 2014;93(17):298-309.

30. Gallagher JC, Kuriakose S, Haynes K, Axelrod P. Case-case-control study of patients with carbapenem-resistant and third-generation-cephalosporinresistant Klebsiella pneumoniae bloodstream infections. Antimicrob Agents Chemother. 2014;58(10):5732-5.

31. Hwee J, Song C, Tan KC, Tan BK, Chong SJ. The trends of burns epidemiology in a tropical regional burns centre. Burns : journal of the International Society for Burn Injuries. 2016;42(3):682-6.

32. Bang RL, Sharma PN, Sanyal SC, Bang S, Ebrahim MK. Burn septicaemia in Kuwait: associated demographic and clinical factors. Med Princ Pract. 2004; 13(3):136-41.

33. Raz-Pasteur A, Hussein K, Finkelstein R, Ullmann Y, Egozi D. Blood stream infections (BSI) in severe burn patients-early and late BSI: a 9-year study. Burns. 2013;39(4):636-42.

34. Cen H, Wu Z, Wang F, Han C. Pathogen distribution and drug resistance in a burn ward: a three-year retrospective analysis of a single center in China. Int J Clin Exp Med. 2015;8(10):19188-99.

35. Shon AS, Bajwa RP, Russo TA. Hypervirulent (hypermucoviscous) Klebsiella pneumoniae: a new and dangerous breed. Virulence. 2013;4(2):107-18.

36. Zhao F, Zhang J, Fu Y, Ruan Z, Xie X. Dissemination of extensively drugresistant and $\mathrm{KPC}-2$ producing Klebsiella pneumoniae isolated from bloodstream infections. J Infect Dev Ctries. 2015;9(9):1016-21.

37. Qureshi ZA, Paterson DL, Potoski BA, Kilayko MC, Sandovsky G, Sordillo E, Polsky B, Adams-Haduch JM, Doi Y. Treatment outcome of bacteremia due to KPC-producing Klebsiella pneumoniae: superiority of combination antimicrobial regimens. Antimicrob Agents Chemother. 2012;56(4):2108-13.

38. Liu YM, Li BB, Zhang YY, Zhang W, Shen H, Li H, Cao B. Clinical and molecular characteristics of emerging hypervirulent Klebsiella pneumoniae bloodstream infections in mainland China. Antimicrob Agents Chemother. 2014;58(9):5379-85.

39. Chiang TT, Yang YS, Yeh KM, Chiu SK, Wang NC, Lin TY, Huang LY, Chang FY, Siu LK, Lin JC, et al. Quantification and comparison of virulence and characteristics of different variants of carbapenemase-producing Klebsiella pneumoniae clinical isolates from Taiwan and the United States. J Microbiol Immunol Infect. 2016:49(1):83-90.

40. Wei DD, Wan LG, Deng Q, Liu Y. Emergence of KPC-producing Klebsiella pneumoniae hypervirulent clone of capsular serotype K1 that belongs to sequence type 11 in Mainland China. Diagn Microbiol Infect Dis. 2016;85(2):192-4.

41. Pau CK, Ma FF, Ip M, You JH. Characteristics and outcomes of Klebsiella pneumoniae bacteraemia in Hong Kong. Infectious diseases (London, England). 2015;47(5):283-8.

42. Michalopoulos A, Falagas ME, Karatza DC, Alexandropoulou P, Papadakis E, Gregorakos L, Chalevelakis G, Pappas G. Epidemiologic, clinical characteristics, and risk factors for adverse outcome in multiresistant gramnegative primary bacteremia of critically ill patients. Am J Infect Control. 2011;39(5):396-400

43. Chen SJ, Chao TF, Chiang MC, Kuo SC, Chen LY, Chiang DH, Yin T, Chen TL, Fung CP. Predictors of mortality in surgical patients with Acinetobacter baumannii bacteremia. J Microbiol Immunol Infect. 2011;44(3):209-14.

44. Hussein K, Raz-Pasteur A, Finkelstein R, Neuberger A, Shachor-Meyouhas Y, Oren I, Kassis I. Impact of carbapenem resistance on the outcome of patients' hospital-acquired bacteraemia caused by Klebsiella pneumoniae. J Hosp Infect. 2013;83(4):307-13.

45. Timsit JF, Laupland KB. Update on bloodstream infections in ICUs. Curr Opin Crit Care. 2012:18(5):479-86.

46. Shorr AF. Review of studies of the impact on Gram-negative bacterial resistance on outcomes in the intensive care unit. Crit Care Med. 2009; 37(4):1463-9.

47. Pradhan NP, Bhat SM, Ghadage DP. Nosocomial infections in the medical ICU: a retrospective study highlighting their prevalence, microbiological profile and impact on ICU stay and mortality. J Assoc Physicians India. 2014; 62(10):18-21.

\section{Submit your next manuscript to BioMed Central and we will help you at every step:}

- We accept pre-submission inquiries

- Our selector tool helps you to find the most relevant journal

- We provide round the clock customer support

- Convenient online submission

- Thorough peer review

- Inclusion in PubMed and all major indexing services

- Maximum visibility for your research

Submit your manuscript at www.biomedcentral.com/submit
C Biomed Central 